\title{
Temperature and pressure dependences of the laser-induced fluorescence of gas-phase acetone and 3-pentanone
}

\author{
F. Grossmann, P. B. Monkhouse, M. Ridder, V. Sick, J. Wolfrum \\ Physikalisch-Chemisches Institut, Im Neuenheimer Feld 253, D-69120 Heidelberg, Germany \\ (Fax: + 49-6221/56-4255) \\ Received: 27 December 1994/Accepted: 12 September 1995
}

\begin{abstract}
Laser-Induced Fluorescence (LIF) from the $S_{1}$ state of acetone and 3-pentanone was studied as a function of temperature and pressure using excitation at $248 \mathrm{~nm}$. Additionally, LIF of 3-pentanone was investigated using 277 and $312 \mathrm{~nm}$ excitation. Added gases were synthetic air, $\mathrm{O}_{2}$, and $\mathrm{N}_{2}$ respectively, in the range $0-50$ bar. At $383 \mathrm{~K}$ and for excitation at $248 \mathrm{~nm}$, all the chosen collision partners gave an initial enhancement in fluorescence intensity with added gas pressure. Thereafter, the signal intensity remained constant for $\mathrm{N}_{2}$ but decreased markedly for $\mathrm{O}_{2}$. For synthetic air, only a small decrease occurred beyond 25 bar. At longer excitation wavelengths ( 277 and $312 \mathrm{~nm}$ ), the corresponding initial rise in signal with synthetic air pressure was less than that for $248 \mathrm{~nm}$. The temperature dependence of the fluorescence intensity was determined in the range $383-640 \mathrm{~K}$ at a constant pressure of 1 bar synthetic air. For $248 \mathrm{~nm}$ excitation, a marked fall in the fluorescence signal was observed, whereas for $277 \mathrm{~nm}$ excitation the corresponding decrease was only half as strong. By contrast, exciting 3-pentanone at $312 \mathrm{~nm}$, the signal intensity increased markedly in the same temperature range. These results are consistent with the observation of a red shift of the absorption spectra $(\approx 9 \mathrm{~nm})$ over this temperature range. Essentially, the same temperature dependence was obtained at 10 and 20 bar pressure of synthetic air. It is demonstrated that temperatures can be determined from the relative fluorescence intensities following excitation of 3-pentanone at 248 and $312 \mathrm{~nm}$, respectively. This new approach could be of interest as a non-intrusive thermometry method, e.g., for the compression phase in combustion engines.
\end{abstract}

PACS: $33.50 ; 33.20 ; 34.90$

There has been interest in the photophysics of carbonyl compounds for many years for several reasons. First, the use of carbonyls as fluorescent dopants was recently proposed for marking fuels in engine combustion [1-3]; the characterization of the fluorescence of acetone and 3-pentanone for this particular application was the motivation for this study. Second, carbonyls are involved in the combustion of hydrocarbons; their thermal decomposition leads largely to free radicals, which serve as chain starters for chemical reactions. Third, the production of free radicals by photodecomposition of carbonyl compounds is of considerable atmospheric importance for the same reason.

The absorption spectrum of acetone and 3-pentanone in the near $\mathrm{UV}$ is due to the excitation of the $\mathrm{C}=\mathrm{O}$ group, whereby a non-bonding electron of oxygen is raised into the antibonding $\pi_{\mathrm{CO}}^{*}$ molecular orbital. The transition from the ground $\left(S_{0}, n\right)$ state to the first excited $\left(S_{1}, \pi^{*}\right)$ state is thus spin-allowed but orbital-forbidden, so that the absorption is relatively weak $\left(\approx 10^{-20} \mathrm{~cm}^{2}\right)$. Absorption occurs from 220 to $330 \mathrm{~nm}$ with a maximum near $280 \mathrm{~nm}$, fluorescence from 300 to $550 \mathrm{~nm}$ with broad maximum at about $400 \mathrm{~nm}$.

The absorption and emission behaviour of a number of ketones have been studied both in the bulk gas [4] and isolated molecule regimes $[5,6]$. The radiative lifetime for the first singlet state of acetone (isolated molecule regime) is around $2 \mathrm{~ms}$. Many investigations, particularly the earlier ones, were made at total pressures of several torr and above, i.e., in the bulk gas. In this regime, effective lifetimes were found to increase with excitation wavelength, varying from $1.7 \mathrm{~ns}$ at $280 \mathrm{~nm}$ to $2.7 \mathrm{~ns}$ at $315 \mathrm{~nm}$ [4]; the corresponding quantum yields increase from $1 \times 10^{-3}$ to $2 \times 10^{-3}$. For 3 -pentanone, the variation in lifetime given in [4] is smaller, i.e., from 2.1 ns at $250 \mathrm{~nm}$ to $2.6 \mathrm{~ns}$ at $325 \mathrm{~nm}$ and the quantum yield is nearly constant in this wavelength range at $2.2 \times 10^{-3}$. In both cases, the $S_{1}$ state decays almost entirely $(99 \%)$ by intersystem crossing to the triplet state.

In this work, we present results of fluorescence intensity measurements of gas-phase acetone and 3-pentanone for pressures up to 50 bar and in the temperature range $383-650 \mathrm{~K}$. The excitation wavelengths selected were 248,277 and $312 \mathrm{~nm}$, which are on the left, near the centre and on the right of the absorption maximum, respectively. 


\section{Experimental}

Time-integrated (relative intensity) measurements (Fig. 1a) were made in the gas phase on acetone and 3-pentanone in a heatable, high-pressure cell, which could be operated safely up to $650 \mathrm{~K}$ and 50 bar. Because the boiling point of 3-pentanone is $375 \mathrm{~K}$, the cell was operated at a minimum temperature of $383 \mathrm{~K}$. The cell was quadratic in outer form and had a cylindrical probe volume of about $320 \mathrm{~cm}^{3}$. The geometry and dimensions were selected so as to ensure instantaneous mixing of gases (Fig. 1b). The ketone of interest was injected through

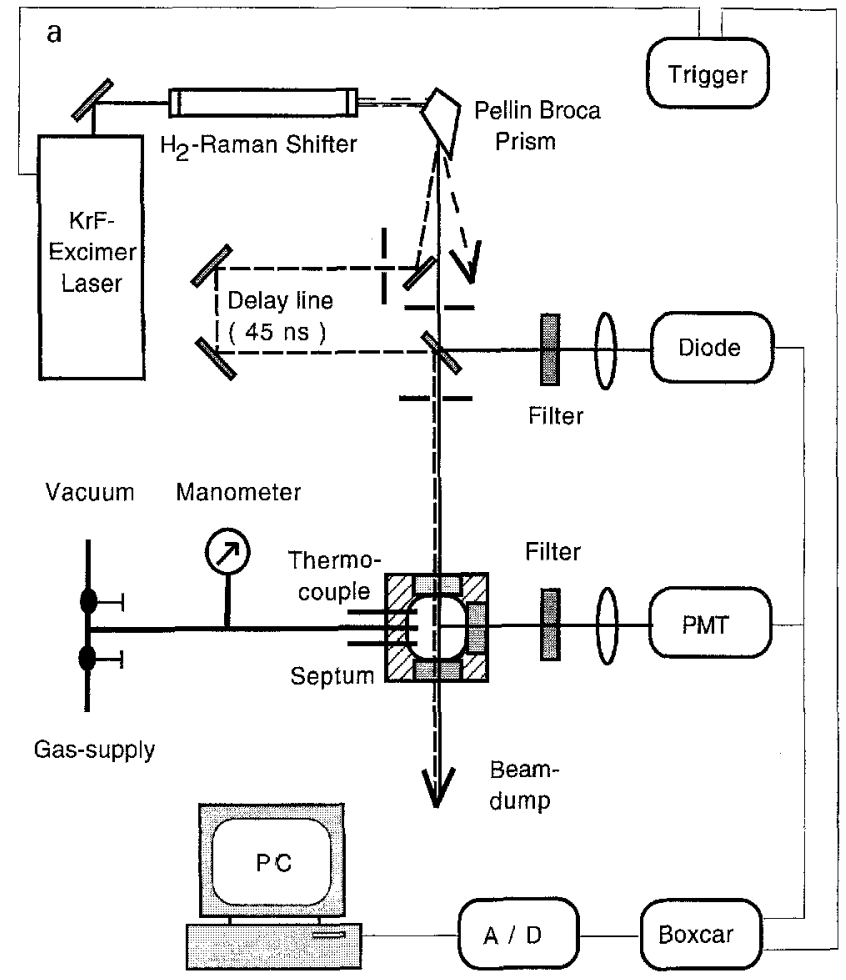

b connection to

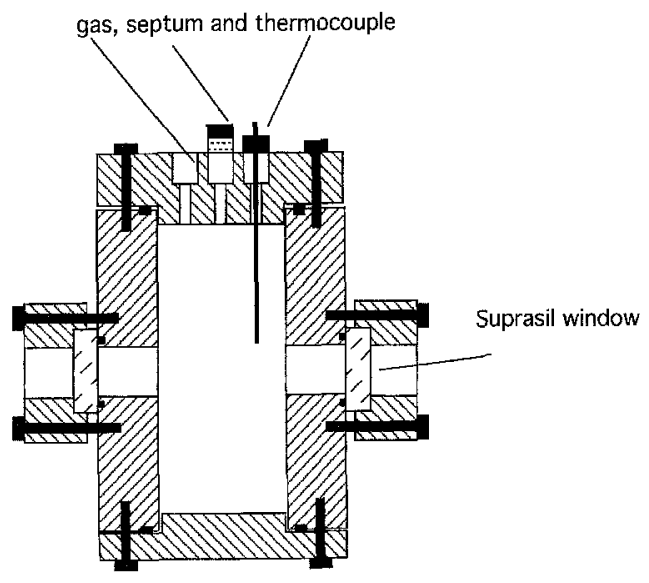

Fig. 1. a Experimental setup for LIF of acetone and 3-pentanone as a function of pressure and temperature. The dashed lines depict the setup used for temperature measurements. b Schematic drawing of the heatable high-pressure cell a septum into the cell and nitrogen, oxygen or synthetic air was added.

For most measurements, a broadband $\mathrm{KrF}$-excimer laser at $248 \mathrm{~nm}$ (Lambda Physik, EMG120ii) was used for excitation and a photomultiplier/boxcar system for detection (Thorn EMI 9783B, Stanford Research Systems 250). Additionally, some measurements were made using 277 and $312 \mathrm{~nm}$ radiation, obtained by Raman shifting the $\mathrm{KrF}$-excimer wavelength (first and second Stokes lines of $\mathrm{H}_{2}$, respectively). Laser intensities of $<1 \mathrm{~mJ} / \mathrm{cm}^{2}$ at a repetition rate of $10 \mathrm{~Hz}$ were used. It was verified that the fluorescence was in the linear regime; neutral density filters were used as required to avoid saturation of the photomultiplier. Approximately 10 laser shots were averaged for each measurement point and the cell was then refilled for the next measurement.

For the temperature measurements by two-line-excitation fluorescence, a slightly modified experimental setup was used. The fluorescence intensity was measured following excitation by two beams ( 248 and $312 \mathrm{~nm}$ ) originating from the same laser pulse: As depicted in Fig. 1a, a PellinBroca prism separated the different wavelengths spatially. The $312 \mathrm{~nm}$ beam was focused directly into the cell, whereas the remaining laser light at $248 \mathrm{~nm}$ was sent through a delay line of approximately $14 \mathrm{~m}$. The fluorescence signals from the two excitations were recorded with the photomultiplier tube (rise time $<3 \mathrm{~ns}$ ) and two gated boxcar-integrators (gate width $15 \mathrm{~ns}$ ).

Recorded emission spectra showed no indication of fragments produced by laser photolysis even at higher intensities. To check for thermal and photolytic decomposition of the investigated ketones, capillary gas chromatography [WCOT-quartz capillary $(51 \mathrm{~m} * 0.32 \mathrm{~mm})$ ] was performed with an iso-thermal programme using a Flame Ionisation Detector (FID). Absorption spectra of the ketones were recorded as a function of temperature using a modified Perkin Elmer, Lambda 7 UVspectrometer.

\section{Results and discussion}

\subsection{Pressure dependence}

All measurements were made using a constant number of molecules $\left(2.8 \times 10^{17} \mathrm{~cm}^{-3}\right.$ for acetone, $2.6 \times 10^{17} \mathrm{~cm}^{-3}$ for 3-pentanone). With an excitation wavelength of $248 \mathrm{~nm}$, a series of fluorescence intensity measurements was made at a constant cell temperature of $383 \mathrm{~K}$, adding nitrogen, oxygen or synthetic air up to total pressures of 30-50 bar. Very similar effects were observed for both acetone and 3-pentanone. As Figs. 2a and b show, the fluorescence intensity rises initially. In the case of $\mathrm{N}_{2}$ (not shown here), the signal levels off when relaxation is complete. This occurred at 5 bar for 3-pentanone and 8 bar for acetone. For $\mathrm{O}_{2}$, after the initial increase, a decrease in signal of $17 \%$ per 10 bar was measured. For synthetic air, on increasing the pressure beyond 25 bar, the signal intensity decreased about $10 \%$ per 10 bar.

The first increase of the signal intensity may be attributed to vibrational relaxation to levels with higher 


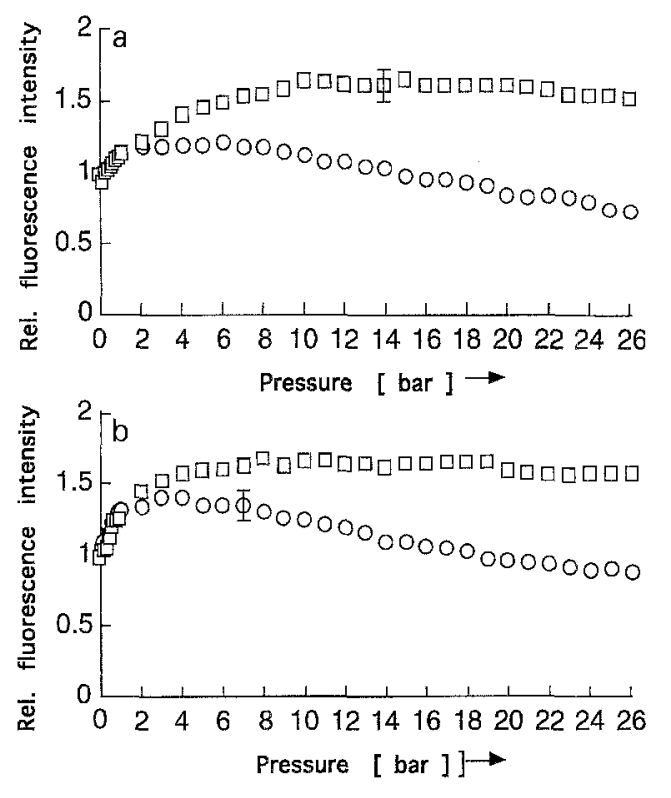

Fig. 2a,b. Pressure dependence of the relative LIF signal for excitation at $248 \mathrm{~nm}$; cell tẹmperature $383 \mathrm{~K}:$ a acetone, b 3-pentanone; (O) oxygen, ( $\square$ ) synthetic air

fluorescence quantum yields. However, quenching of the $S_{1}$ state by $\mathrm{O}_{2}$ is able to compete effectively with this vibrational relaxation. Quenching of excited organic molecules by ground-state (triplet) oxygen is well known $[7,8]$ and in this case may be due to the formation of a chargetransfer complex with the ketone, involving the electronegative oxygen atom of the excited carbonyl bond and electrons of the double bond. From this model one expects no change in the lifetime of the $S_{1}$ state in the presence of air or oxygen as opposed to nitrogen, as confirmed by Ossler and Alden [9], who obtained the same lifetime $(2.6 \pm 0.25 \mathrm{~ns})$ for 3-pentanone in 9.2 bar nitrogen and air at $523 \mathrm{~K}$. A similar result was obtained with aldehydes [10].

Two further series of experiments were conducted for excitation of 3-pentanone at 277 and $312 \mathrm{~nm}$. At $277 \mathrm{~nm}$, the fluorescence intensity increased slightly with synthetic air pressure up to 2 bar and then decreased approximately $40 \%$ at 50 bar. For $312 \mathrm{~nm}$ excitation, almost no initial increase in signal intensity is measured. Similar results were obtained at 473 and $533 \mathrm{~K}$. Figure 3 shows the difference of the relative fluorescence intensity at $473 \mathrm{~K}$ using excitation at 248 and $312 \mathrm{~nm}$. The longer wavelength at $312 \mathrm{~nm}$ is very close to the lowest vibrational level of the $S_{1}$ state of the ketone. Then, no or little vibrational relaxation takes place and, therefore, the quenching effect is observable at much lower pressures.

Because of the interest in ketones for engine diagnostics, the pressure dependence of 3-pentanone fluorescence on synthetic air pressure was also investigated using a mixture of iso-octane and 3-pentanone $(80: 20 \%)$ and $248 \mathrm{~nm}$ excitation. Again, an initial increase in signal intensity was observed up to 5 bar, followed by an almost constant intensity up to 25 bar.

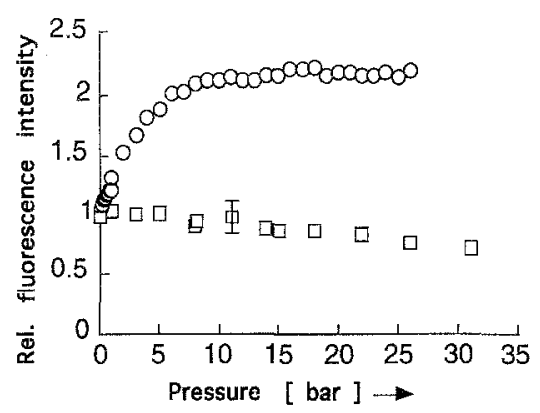

Fig. 3. Pressure dependence of the relative LIF signal of 3-pentanone. $\lambda_{\mathrm{ex}}: 248 \mathrm{~nm}(\mathrm{O})$ and $312 \mathrm{~nm}(\square)$ at $473 \mathrm{~K}$

\subsection{Temperature dependence}

Relative intensity measurements were made for both acetone and 3-pentanone at a constant pressure of 1 bar synthetic air in the temperature range $383-573 \mathrm{~K}$. Using $248 \mathrm{~nm}$ as the excitation wavelength, the signal decreased as shown in Fig. 4 (approximately $35 \%$ per $100 \mathrm{~K}$ ). To establish whether this effect was due to the decomposition of the fluorescing molecule, gas chromatographic measurements were made by sampling from the cell via a septum. Both the ketone and its decomposition products (ethylene, butenes and a small amount of propionaldehyde) were monitored. The results showed that even after $10 \mathrm{~min}$ residence time in the cell at $575 \mathrm{~K}$, only $10 \%$ of the injected compound had dissociated. Thus, the observed fall in fluorescence intensity (measured immediately after injection) cannot be due to decomposition.

For further clarification, absorption spectra of acetone and 3-pentanone were recorded as a function of temperature in the range $298-573 \mathrm{~K}$; Fig. 5 shows the results for 3-pentanone. The absorption peak at $275 \mathrm{~nm}$ shifts to longer wavelengths with increasing temperature, while the halfwidth remains almost constant (approximately $30 \mathrm{~nm}$ ). This means that the absorption strength will decrease at $248 \mathrm{~nm}$ (on the left of the absorption maximum) and a decrease in fluorescence signal intensity is to be expected using this excitation wavelength, as observed. A smaller temperature dependence at $277 \mathrm{~nm}$ is expected since this wavelength is close to the absorption maximum. Accordingly, if this ketone is excited on the right-hand side of the absorption maximum, a positive temperature dependence should be measured, as observed by Greenhalgh and Tait [11] for $308 \mathrm{~nm}$.

The temperature dependence of 3-pentanone was also determined using 277 and $312 \mathrm{~nm}$ excitation (Fig. 4). In the temperature range $383-573 \mathrm{~K}$, the fluorescence intensity decreased by $15 \%$ per $100 \mathrm{~K}$ for $277 \mathrm{~nm}$ excitation, but increased about $80 \%$ for $312 \mathrm{~nm}$ excitation. For $277 \mathrm{~nm}$ excitation the same measurements were performed at 1,10 and 20 bar (synthetic air), the observed temperature dependence was essentially the same at all three pressures (Fig. 6). Tait and Greenhalgh [11] investigated the temperature dependence of several carbonyl compounds at atmospheric pressure in a flow system. The 


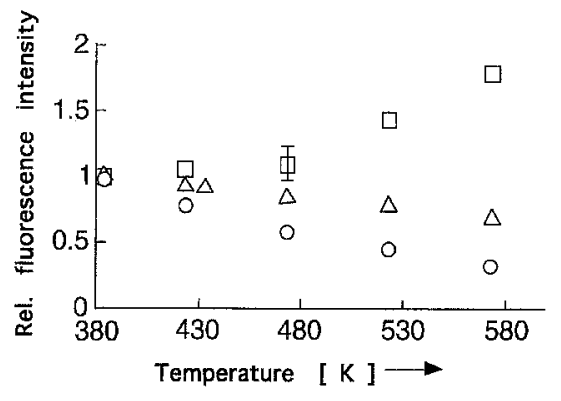

Fig. 4. Temperature dependence of the relative LIF signal for 3pentanone for excitation at $248 \mathrm{~nm}(\bigcirc), 277 \mathrm{~nm}(\triangle)$ and $312 \mathrm{~nm}(\square)$. All data are normalized with respect to the signal intensities at $383 \mathrm{~K}$

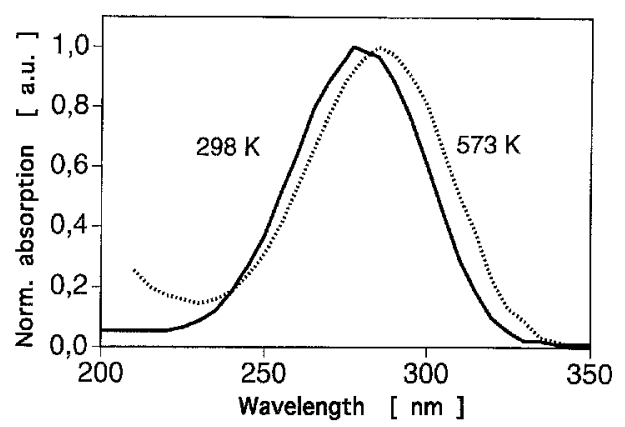

Fig. 5. Absorption spectrum of 3-pentanone at different temperatures and atmospheric pressure. $298 \mathrm{~K}$ (full line) and $573 \mathrm{~K}$ (dotted line)

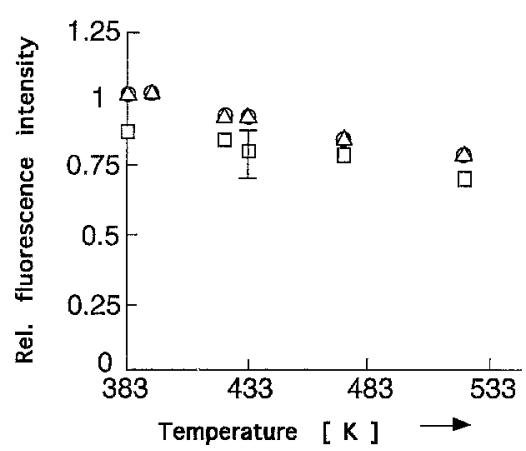

Fig. 6. Temperature dependence of the relative LIF signal for 3pentanone. $\lambda_{\mathrm{ex}}=277 \mathrm{~nm}$, at 1 bar $(O), 10 \mathrm{bar}(\triangle)$ and 20 bar $(\square)$ synthetic air pressure

excitation wavelength there was $308 \mathrm{~nm}$ (XeCl-excimer) and the temperature range studied was $300-800 \mathrm{~K}$. For acetone and 3-pentanone, a steady increase in signal of $16 \%$ per $100 \mathrm{~K}$ was observed.

\subsection{Temperature measurement using two-line-excitation technique of 3-pentanone}

The temperature dependence of the absorption strength - and hence the fluorescence intensity - of ketones offers the possibility to determine the temperature. To

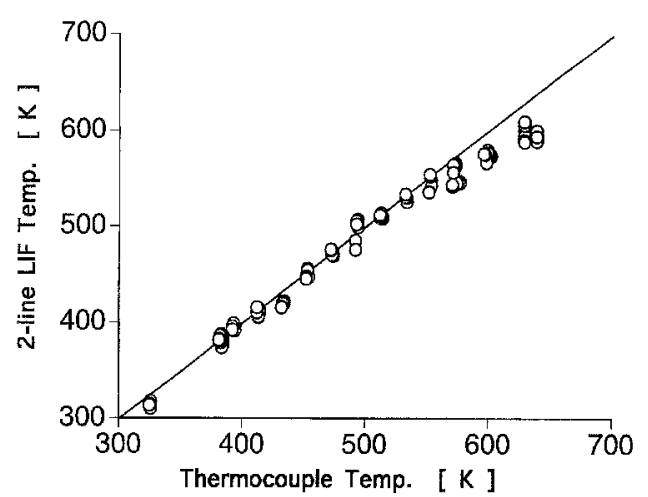

Fig. 7. Temperature measurement using two-line fluorescence of 3-pentanone. The calculated temperature from these measurements is shown as a function of the corresponding thermocouple readings

demonstrate the possibilities of this new approach, we measured the ratio of the fluorescence intensity following 248 and $312 \mathrm{~nm}$ excitations.

Measurements were performed at atmospheric pressure (synthetic air) in the temperature range $350-640 \mathrm{~K}$. Again, the fluorescence intensities were measured as a function of temperature and all data were normalized with respect to the signal strengths at $383 \mathrm{~K}$. For each pair of excitation pulses the ratio $I_{312 \mathrm{~nm}}(T) / I_{248 \mathrm{~nm}}(T)$ was then calculated. From the results of the measurements mentioned before (Fig. 4), we determined the relative fluorescence intensity as a function of temperature and used this calibration curve to calculate temperature from the measured ratio. Figure 7 shows the comparison of the calculated temperature and the thermocouple readings. Each measurement point corresponds to the mean value of 15 successive laser pulses. Within the whole temperature range investigated here, there is good agreement between the calculated and the measured temperatures. However, at the highest temperatures, the calculated values tend to underestimate the thermocouple readings by $40-50 \mathrm{~K}$. The mean scatter within a series for a given temperature was $\pm 21 \mathrm{~K}$.

\section{Conclusions}

Over a wide pressure range (ca. 5-25 bar), the fluorescence intensity of both acetone and 3-pentanone are constant. The temperature dependence of the fluorescence is dependent on the excitation wavelength and can be minimized near the absorption maximum. These results show that these ketones will be suitable fluorescent dopants for marking fuel distributions in combustors and fast-flow devices. 3-pentanone can be used with common model fuels, such as iso-octane [2,3], because of similar boiling points and mass diffusion coefficients. These ketones are chemically relatively stable and only dissociate at higher temperatures (i.e., in the flame front).

It is demonstrated that two-line fluorescence of 3-pentanone is a new optical technique for non-intrusive thermometry. Since there is only little influence of pressure on 
the ratio of the fluorescence intensities of two different excitation wavelengths, this new approach could be of special interest to applications operating at elevated pressures, e.g., in the compression stroke of Diesel engines. To improve the signal-to-noise ratio, i.e., to compensate for laser fluctuations or any other temporal drift effects, the laser power should be measured online before as well after the pressure vessel. Whereas the precision of this approach is quite satisfactory up to $550 \mathrm{~K}$, the deviation at higher temperatures may be due to uncertainties of the calibration curve above $600 \mathrm{~K}$; this will be investigated in further work.

Acknowledgements. This work was supported by the Bundesministerium für Forschung und Technologie (Arbeitsgemeinschaft TECFLAM) and the Commission of the European Union (Project MIDCOM II)

\section{References}

1. A. Arnold, H. Becker, R. Suntz, P. Monkhouse, J. Wolfrum, R. Maly, W. Pfister: Opt. Lett. 15, 831 (1989)

2. A. Arnold, A. Buschmann, B. Cousyn, M. Decker, F. Vannobel, V. Sick, J. Wolfrum: SAE Paper 932696 (1993)

3. H. Neij, M. Aldén: Combust. Flame 99, 449 (1994)

4. D.A. Hansen, E.K.C. Lee: J. Chem. Phys. 62, 183 (1975)

5. A. Lozano, B. Yip, R.K. Hanson: Exp. Fluids 13, 369 (1992)

6. Y. Haas: Spectrochim. Acta A 46, 541 (1990)

7. O.L. Gijzmann: Faraday Trans. II 70, 708 (1973)

8. P.B. Merkel, D.J. Kearns: J. Chem. Phys. 58, 398 (1973)

9. F. Ossler, M. Aldén: Appl. Spectrosc. (1996) (submitted)

10. A. Arnold, A. Bräumer, F. Dinkelacker, P. Monkhonse, K. Witte, M. Schäfer, M. Köllner, W. Ketterle, J. Wolfrum: Final Report, CEC-Project JOULE0021-UK/JC, European Commission (1992)

11. R. Tait, D. Greenhalgh: Ber Bunsenges. Phys. Chem. 97, 1619 (1993) 